\title{
Subclasses of analytic functions of complex order defined by $q$-derivative operator
}

\author{
Rekha Srivastava and Hanaa M. Zayed
}

\begin{abstract}
Using the $q$-derivative operator in conjunction with the principle of subordination between analytic functions, we introduce two subclasses of analytic functions in the open unit disk $\mathbb{U}$. We investigate convolution properties and coefficient estimates for these subclasses.
\end{abstract}

Mathematics Subject Classification (2010): 30C45, 30C50.

Keywords: Analytic functions, univalent functions, Hadamard product (or convolution), subordination between analytic functions, $q$-derivative operator.

\section{Introduction}

Recently, the theory of $q$-analysis has attracted a considerable effort of researchers due to its application in many branches of mathematics and physics, for example, in the areas of ordinary fractional calculus, optimal control problems, $q$-difference, $q$ integral equations and in $q$-transform analysis (see for instance $[1,9,11,19]$ ). The main purpose of this paper is to introduce and study two subclasses of analytic functions in the open unit disk

$$
\mathbb{U}=\{z: z \in \mathbb{C} \text { and }|z|<1\}
$$

by applying the $q$-derivative operator in conjunction with the principle of subordination between analytic functions.

Let $\mathcal{A}$ denote the class of functions $f(z)$ of the form:

$$
f(z)=z+\sum_{k=2}^{\infty} a_{k} z^{k},
$$

which are analytic in $\mathbb{U}$. Also $\mathcal{S}$ be the subclass of all functions in $\mathcal{A}$, which are univalent in $\mathbb{U}$. Let $\mathcal{S}^{*}(\alpha)$ and $\mathcal{K}(\alpha)$ denote the subclasses of $\mathcal{S}$ consisting of starlike and convex functions of order $\alpha(0 \leqq \alpha<1)$. We note that

$$
\mathcal{S}^{*}(0)=\mathcal{S}^{*} \quad \text { and } \quad \mathcal{K}(0)=\mathcal{K},
$$


where $\mathcal{S}^{*}$ and $\mathcal{K}$ denote, respectively, the familiar subclasses of starlike and convex functions (see, for details, Srivastava and Owa [25]).

Let $\mathcal{K}[b ; A, B]$ and $\mathcal{S}[b ; A, B]\left(b \in \mathbb{C}^{*}=\mathbb{C} \backslash\{0\},-1 \leqq B<A \leqq 1, z \in \mathbb{U}\right)$ denote the subclasses of $\mathcal{A}$ and satisfy the following conditions:

$$
\mathcal{K}[b ; A, B]=\left\{f: f(z) \in \mathcal{A} \quad \text { and } \quad 1+\frac{1}{b} \frac{z f^{\prime \prime}(z)}{f^{\prime}(z)} \prec \frac{1+A z}{1+B z}\right\}
$$

and

$$
\mathcal{S}[b ; A, B]=\left\{f: f(z) \in \mathcal{A} \quad \text { and } \quad 1+\frac{1}{b}\left[\frac{z f^{\prime}(z)}{f(z)}-1\right] \prec \frac{1+A z}{1+B z}\right\},
$$

where the symbol $\prec$ stands for subordination between analytic functions (see [13]) (see also [5] and [23]). The class $\mathcal{K}[b ; A, B]$ was introduced and studied by Aouf et al. $[3]$ and the class $\mathcal{S}[b ; A, B]$ was introduced and studied by Sohi and Singh [21] (see also Aouf et al. [3] and [4]).

We note that

(i) $\mathcal{K}[b ; 1,-1]=\mathcal{C}(b)$ (see Nasr and Aouf [15]).

(ii) $\mathcal{S}[b ; 1,-1]=\mathcal{S}(b)$ (see Nasr and Aouf [18]).

For $f(z) \in \mathcal{A}$, the $q$-derivative $(0<q<1)$ of $f(z)$ is defined by (see Gasper and Rahman [9])

$$
D_{q} f(z)= \begin{cases}\frac{f(q z)-f(z)}{(q-1) z} & (z \neq 0) \\ f^{\prime}(0) & (z=0),\end{cases}
$$

provided that $f^{\prime}(0)$ exists. From (1.2), we deduce that

$$
D_{q} f(z)=1+\sum_{k=2}^{\infty}[k]_{q} a_{k} z^{k-1} \quad(z \neq 0)
$$

where

$$
[k]_{q}=\frac{1-q^{k}}{1-q}
$$

As $q \rightarrow 1^{-},[k]_{q} \rightarrow k$ and

$$
\lim _{q \rightarrow 1^{-}} D_{q} f(z)=f^{\prime}(z) .
$$

Also, the $q$-integral of a function $f(z)$ is defined by (see Gasper and Rahman [9])

$$
\int_{0}^{z} f(t) d_{q} t=z(1-q) \sum_{k=0}^{\infty} q^{k} f\left(z q^{k}\right) .
$$

It should be observed here that, as already pointed out by Srivastava and Bansal $[24$, p. 62$]$, although the $q$-derivative operator in (1.2) was first applied to study a $q$-extension of the class $\mathcal{S}^{*}$ of starlike functions in $\mathbb{U}$, a firm footing of the usage of the $q$-calculus in the context of Geometric Function Theory was actually provided and the basic (or $q$-) hypergeometric functions were first used in Geometric Function Theory in a book chapter by Srivastava (see, for details, [22, pp. 347 et seq.]). 
Making use of the $q$-derivative $D_{q}$ given by (1.2), we introduce $\mathcal{K}_{q}[b ; A, B]$ and $\mathcal{S}_{q}[b ; A, B]$ of $\mathcal{A}$ for $b \in \mathbb{C}^{*}, 0<q<1$ and $-1 \leqq B<A \leqq 1$ as follows:

$$
\mathcal{K}_{q}[b ; A, B]=\left\{f(z) \in \mathcal{A}: 1+\frac{1}{b}\left[\frac{D_{q}\left(z D_{q} f(z)\right)}{D_{q} f(z)}-1\right] \prec \frac{1+A z}{1+B z}\right\},
$$

and

$$
\mathcal{S}_{q}[b ; A, B]=\left\{f(z) \in \mathcal{A}: 1+\frac{1}{b}\left[\frac{z D_{q} f(z)}{f(z)}-1\right] \prec \frac{1+A z}{1+B z}\right\} .
$$

From (1.4) and (1.5), we find that

$$
f(z) \in \mathcal{S}_{q}[b ; A, B] \Longleftrightarrow \int_{0}^{z} \frac{f(\zeta)}{\zeta} d_{q} \zeta \in \mathcal{K}_{q}[b ; A, B] .
$$

We also note that

(i) $\mathcal{K}_{q}[1 ; A, B]=\mathcal{K}_{q}[A, B]$ and $\mathcal{S}_{q}[1 ; A, B]=\mathcal{S}_{q}[A, B]$ (see Seoudy and Aouf [20]);

(ii) $\lim _{q \rightarrow 1^{-}} \mathcal{K}_{q}[b ; A, B]=\mathcal{K}[b ; A, B]$ (see Aouf et al. [3]) and

$\lim _{q \rightarrow 1^{-}} \mathcal{S}_{q}[b ; A, B]=\mathcal{S}[b ; A, B]$ (see Sohi and Singh [21]) (see also Aouf et al. [3] and [4]);

(iii) $\mathcal{K}_{q}\left[b ; 1, \frac{1-M}{M}\right]=\mathcal{G}_{q}(b, M)$

$$
=\left\{f(z) \in \mathcal{A}:\left|\frac{b-1+\frac{D_{q}\left(z D_{q} f(z)\right)}{D_{q} f(z)}}{b}-M\right|<M\left(M>\frac{1}{2}\right)\right\} ;
$$

and $\mathcal{S}_{q}\left[b ; 1, \frac{1-M}{M}\right]=\mathcal{F}_{q}(b, M)$

$$
=\left\{f(z) \in \mathcal{A}:\left|\frac{b-1+\frac{z D_{q} f(z)}{f(z)}}{b}-M\right|<M\left(M>\frac{1}{2}\right)\right\} ;
$$

(iv) $\lim _{q \rightarrow 1^{-}} \mathcal{K}_{q}\left[b ; 1, \frac{1-M}{M}\right]=\lim _{q \rightarrow 1^{-}} \mathcal{G}_{q}(b, M)=\mathcal{G}(b, M)$ (see Nasr and Aouf [17]) and $\lim _{q \rightarrow 1^{-}} \mathcal{S}_{q}\left[b ; 1, \frac{1-M}{M}\right]=\lim _{q \rightarrow 1^{-}} \mathcal{F}_{q}(b, M)=\mathcal{F}(b, M)$ (see Nasr and Aouf [16]);

(v) $\mathcal{G}_{q}\left(1-m-M, \frac{M}{m+M-1}\right)=\mathcal{C}_{q}(m, M)$

$$
=\left\{f(z) \in \mathcal{A}:\left|\frac{D_{q}\left(z D_{q} f(z)\right)}{D_{q} f(z)}-m\right|<M\left(m=1-\frac{1}{M} ; M>\frac{1}{2}\right)\right\} ;
$$

and $\mathcal{F}_{q}\left(1-m-M, \frac{M}{m+M-1}\right)=\mathfrak{B}_{q}(m, M)$

$$
=\left\{f(z) \in \mathcal{A}:\left|\frac{z D_{q} f(z)}{f(z)}-m\right|<M\left(m=1-\frac{1}{M} ; M>\frac{1}{2}\right)\right\} ;
$$

(vi) $\lim _{q \rightarrow 1^{-}} \mathfrak{B}_{q}(m, M)=\mathfrak{B}(m, M)$ (see Jakubowski [10]);

(vii) $\mathcal{K}_{q}[b ; 1,-1]=\mathcal{K}_{q}(b)$

$$
=\left\{f(z) \in \mathcal{A}: \operatorname{Re}\left(1+\frac{1}{b}\left[\frac{D_{q}\left(z D_{q} f(z)\right)}{D_{q} f(z)}-1\right]\right)>0(z \in \mathbb{U})\right\} ;
$$


and $\mathcal{S}_{q}[b ; 1,-1]=\mathcal{S}_{q}(b)$

$$
=\left\{f(z) \in \mathcal{A}: \operatorname{Re}\left(1+\frac{1}{b}\left[\frac{z D_{q} f(z)}{f(z)}-1\right]\right)>0(z \in \mathbb{U})\right\} ;
$$

(viii) $\lim _{q \rightarrow 1^{-}} \mathcal{K}_{q}[b ; 1,-1]=\lim _{q \rightarrow 1^{-}} \mathcal{C}_{q}(b)=\mathcal{C}(b)$ (see Nasr and Aouf [15]) and $\lim _{q \rightarrow 1^{-}} \mathcal{S}_{q}[b ; 1,-1]=\lim _{q \rightarrow 1^{-}} \mathcal{S}_{q}(b)=\mathcal{S}(b)$ (see Nasr and Aouf [18]);

(ix) $\mathcal{K}_{q}\left[e^{-i \lambda} \cos \lambda ; A, B\right]=\mathcal{K}_{q}^{\lambda}[A, B]$

$$
=\left\{f(z) \in \mathcal{A}: e^{i \lambda} \frac{D_{q}\left(z D_{q} f(z)\right)}{D_{q} f(z)} \prec \cos \lambda \frac{1+A z}{1+B z}+i \sin \lambda\left(|\lambda|<\frac{\pi}{2}\right)\right\} ;
$$

and $\mathcal{S}_{q}\left[e^{-i \lambda} \cos \lambda ; A, B\right]=\mathcal{S}_{q}^{\lambda}[A, B]$

$$
=\left\{f(z) \in \mathcal{A}: e^{i \lambda} \frac{z D_{q} f(z)}{f(z)} \prec \cos \lambda \frac{1+A z}{1+B z}+i \sin \lambda\left(|\lambda|<\frac{\pi}{2}\right)\right\} ;
$$

(x) $\lim _{q \rightarrow 1^{-}} \mathcal{K}_{q}\left[e^{-i \lambda} \cos \lambda ; A, B\right]=\mathcal{K}^{\lambda}[A, B] \quad\left(|\lambda|<\frac{\pi}{2}\right)$ (see Bhoosnurmath and Devadas [7]) and $\lim _{q \rightarrow 1^{-}} \mathcal{S}_{q}\left[e^{-i \lambda} \cos \lambda ; A, B\right]=\mathcal{S}^{\lambda}[A, B]\left(|\lambda|<\frac{\pi}{2}\right)$ (see Dashrath and Shukla [8]) (see Bhoosnurmath and Devadas [6]; see also the more recent work by Xu et al. [26]); (xi) $\mathcal{K}_{q}\left[e^{-i \lambda} \cos \lambda ; A, B\right]=\mathcal{G}_{q, \lambda, M}$

$$
=\left\{f(z) \in \mathcal{A}:\left|\frac{e^{i \lambda \frac{D_{q}\left(z D_{q} f(z)\right)}{D_{q} f(z)}-i \sin \lambda}}{\cos \lambda}-M\right|<M \quad\left(|\lambda|<\frac{\pi}{2} ; M>\frac{1}{2}\right)\right\}
$$

and $\mathcal{S}_{q}\left[e^{-i \lambda} \cos \lambda ; 1, \frac{1-M}{M}\right]=\mathcal{F}_{q, \lambda, M}$

$$
=\left\{f(z) \in \mathcal{A}:\left|\frac{e^{i \lambda \frac{z D_{q} f(z)}{f(z)}-i \sin \lambda}}{\cos \lambda}-M\right|<M\left(|\lambda|<\frac{\pi}{2} ; M>\frac{1}{2}\right)\right\}
$$

(xii) $\lim _{q \rightarrow 1^{-}} \mathcal{K}_{q}\left[e^{-i \lambda} \cos \lambda ; 1, \frac{1-M}{M}\right]=\lim _{q \rightarrow 1^{-}} \mathcal{G}_{q, \lambda, M}=\mathcal{G}_{\lambda, M}$ and $\lim _{q \rightarrow 1^{-}} \mathcal{S}_{q}\left[e^{-i \lambda} \cos \lambda ; 1, \frac{1-M}{M}\right]=\lim _{q \rightarrow 1^{-}} \mathcal{F}_{q, \lambda, M}=\mathcal{F}_{\lambda, M}$ (see Kulshrestha $[12]$ ); (xiii) $\mathcal{K}_{q}\left[(1-\mu) e^{-i \lambda} \cos \lambda ; 1, \frac{1-M}{M}\right]=\mathcal{G}_{q}[\lambda, \mu, M]$

$$
\begin{aligned}
= & \left\{f(z) \in \mathcal{A}:\left|\frac{e^{i \lambda} \frac{D_{q}\left(z D_{q} f(z)\right)}{D_{q} f(z)}-\mu \cos \lambda-i \sin \lambda}{(1-\mu) \cos \lambda}-M\right|<M\right. \\
& \left.\left(|\lambda|<\frac{\pi}{2} ; 0 \leqq \mu<1 ; M>\frac{1}{2}\right)\right\} ;
\end{aligned}
$$


and $\mathcal{S}_{q}\left[(1-\mu) e^{-i \lambda} \cos \lambda ; 1, \frac{1-M}{M}\right]=\mathcal{F}_{q}[\lambda, \mu, M]$

$$
\begin{aligned}
= & \left\{f(z) \in \mathcal{A}:\left|\frac{e^{i \lambda \frac{z D_{q} f(z)}{f(z)}-\mu \cos \lambda-i \sin \lambda}}{(1-\mu) \cos \lambda}-M\right|<M\right. \\
& \left.\left(|\lambda|<\frac{\pi}{2} ; 0 \leqq \mu<1 ; M>\frac{1}{2}\right)\right\} ;
\end{aligned}
$$

(xiv) $\lim _{q \rightarrow 1^{-}} \mathcal{K}_{q}\left[(1-\mu) e^{-i \lambda} \cos \lambda ; 1, \frac{1-M}{M}\right]=\lim _{q \rightarrow 1^{-}} \mathcal{K}_{q}[\lambda, \mu, M]=\mathcal{K}[\lambda, \mu, M]$ and $\lim _{q \rightarrow 1^{-}} \mathcal{S}_{q}\left[(1-\mu) e^{-i \lambda} \cos \lambda ; 1, \frac{1-M}{M}\right]=\lim _{q \rightarrow 1^{-}} \mathcal{F}_{q}[\lambda, \mu, M]=\mathcal{F}[\lambda, \mu, M]$ (see Aouf [2]).

\section{Main results}

Unless otherwise mentioned, we assume throughout this paper that $0<q<1$, $-1 \leq B<A \leq 1, b \in \mathbb{C}^{*}, z \in \mathbb{U}$ and $\theta \in[0,2 \pi)$.

Theorem 2.1. If $f(z) \in \mathcal{A}$, then $f(z) \in \mathcal{K}_{q}[b ; A, B]$ if and only if

$$
\frac{1}{z}\left[f(z) * \frac{z+[1-(1+M(\theta))(q+1)] q z^{2}}{(1-z)(1-q z)\left(1-q^{2} z\right)}\right] \neq 0,
$$

where the symbol * stands for the convolution between two power series and

$$
M(\theta)=M^{b ; A, B}(\theta)=\frac{1}{b}\left(\frac{e^{-i \theta}+B}{A-B}\right) .
$$

Proof. It is easy to verify that

$$
z D_{q} f(z) * \frac{z}{1-z}=z D_{q} f(z) \text { and } z D_{q} f(z) * \frac{z}{(1-z)(1-q z)}=z D_{q}\left(z D_{q} f(z)\right) .
$$

In order to prove that (2.1) holds we will write (1.4) by using the definition of the subordination, that is

$$
1+\frac{1}{b}\left[\frac{D_{q}\left(z D_{q} f(z)\right)}{D_{q} f(z)}-1\right]=\frac{1+A w(z)}{1+B w(z)},
$$

where $w(z)$ is Schwarz function, hence

$$
\frac{1}{z}\left[z D_{q}\left(z D_{q} f(z)\right)\left(1+B e^{i \theta}\right)-\left[1+[B+b(A-B)] e^{i \theta}\right] z D_{q} f(z)\right] \neq 0 .
$$

Using (2.3), Eq. (2.5) may be written as

$$
\begin{gathered}
\frac{1}{z}\left[\left(1+B e^{i \theta}\right)\left(z D_{q} f(z) * \frac{z}{(1-z)(1-q z)}\right)\right. \\
\left.-\left[1+[B+b(A-B)] e^{i \theta}\right]\left(z D_{q} f(z) * \frac{z}{1-z}\right)\right] \neq 0,
\end{gathered}
$$


which is equivalent to

or

$$
\frac{1}{z}\left[z D_{q} f(z) * \frac{z-\left(1+\frac{e^{-i \theta}+B}{(A-B) b}\right) q z^{2}}{(1-z)(1-q z)} \cdot\left[-(A-B) b e^{i \theta}\right]\right] \neq 0
$$

$$
\begin{gathered}
\frac{1}{z}\left[f(z) * z D_{q} \frac{z-\left(1+\frac{e^{-i \theta}+B}{(A-B) b}\right) q z^{2}}{(1-z)(1-q z)}\right] \\
=\frac{1}{z}\left[f(z) * \frac{z+\left[1-(q+1)\left(1+\frac{e^{-i \theta}+B}{(A-B) b}\right)\right] q z^{2}}{(1-z)(1-q z)\left(1-q^{2} z\right)}\right] \neq 0,
\end{gathered}
$$

that is (2.1). Reversely, since, it was shown in the first part of the proof that the assumption (2.5) is equivalent to (2.1), we obtain that

$$
\frac{D_{q}\left(z D_{q} f(z)\right)}{D_{q} f(z)} \neq \frac{1+[B+(A-B) b] e^{i \theta}}{1+B e^{i \theta}} .
$$

Suppose that

$$
\varphi(z)=\frac{D_{q}\left(z D_{q} f(z)\right)}{D_{q} f(z)} \quad \text { and } \quad \psi(z)=\frac{1+[B+(A-B) b] z}{1+B z} .
$$

The relation (2.6) means that

$$
\varphi(\mathbb{U}) \cap \psi(\partial \mathbb{U})=\emptyset .
$$

Thus, the simply connected domain is included in a connected component of $\mathbb{C} \backslash \psi(\partial \mathbb{U})$. From this, using the fact that $\varphi(0)=\psi(0)$ and the univalence of the function $\psi$, it follows that $\varphi(z) \prec \psi(z)$, this implies that $f(z) \in \mathcal{K}_{q}[b ; A, B]$. Thus, the proof is completed.

Theorem 2.2. If $f(z) \in \mathcal{A}$, then $f(z) \in \mathcal{S}_{q}[b ; A, B]$ if and only if

$$
\frac{1}{z}\left[f(z) * \frac{z-(1+M(\theta)) q z^{2}}{(1-z)(1-q z)}\right] \neq 0,
$$

where $M(\theta)$ is given by (2.2).

Proof. From (1.6), it follows that $f \in \mathcal{S}_{q}[b ; A, B]$ if and only if

$$
\Phi_{q}(z)=\int_{0}^{z} \frac{f(\zeta)}{\zeta} d_{q} \zeta \in \mathcal{K}_{q}[b ; A, B]
$$

Then, according to Theorem 2.1, the function $\Phi_{q}$ belongs to $\mathcal{S}_{q}[b ; A, B]$ if and only if

$$
\frac{1}{z}\left[\Phi_{q}(z) * g(z)\right] \neq 0, \text { for all } z \in \mathbb{U} \text { and } \theta \in[0,2 \pi),
$$


where

$$
g(z)=\frac{z+[1-(1+M(\theta))(q+1)] q z^{2}}{(1-z)(1-q z)\left(1-q^{2} z\right)} .
$$

From (1.3), we have

$$
\begin{aligned}
\int_{0}^{z} \frac{g(\zeta)}{\zeta} d_{q} \zeta & =\int_{0}^{z} \frac{1+[1-(1+M(\theta))(q+1)] q \zeta}{(1-\zeta)(1-q \zeta)\left(1-q^{2} \zeta\right)} d_{q} \zeta \\
& =z(1-q) \sum_{k=0}^{\infty} \frac{q^{k}+[1-(1+M(\theta))(q+1)] z q^{2 k+1}}{\left(1-z q^{k}\right)\left(1-z q^{k+1}\right)\left(1-z q^{k+2}\right)}
\end{aligned}
$$

and therefore

$$
\int_{0}^{z} \frac{g(\zeta)}{\zeta} d_{q} \zeta=\frac{z-(1+M(\theta)) q z^{2}}{(1-z)(1-q z)}
$$

Using the above relation and the identity

$$
\left[\int_{0}^{z} \frac{f(\zeta)}{\zeta} d_{q} \zeta\right] * g(z)=f(z) *\left[\int_{0}^{z} \frac{g(\zeta)}{\zeta} d_{q} \zeta\right]
$$

it is easy to check that (2.8) is equivalent to (2.7).

Theorem 2.3. If $f(z) \in \mathcal{A}$, then $f(z) \in \mathcal{K}_{q}[b ; A, B]$ if and only if

$$
1-\sum_{k=2}^{\infty}[k]_{q} \frac{\left([k]_{q}-1\right)\left(e^{-i \theta}+B\right)-(A-B) b}{(A-B) b} a_{k} z^{k-1} \neq 0 \text { for all } \theta .
$$

Proof. If $f(z) \in \mathcal{A}$, then from Theorem 2.1, we have $f(z) \in \mathcal{K}_{q}[b ; A, B]$ if and only if (2.1) holds. Since

$$
\begin{aligned}
\frac{1}{(1-z)(1-q z)\left(1-q^{2} z\right)} & =1+\left(1+q+q^{2}\right) z+\left(1+q+2 q^{2}+q^{3}+q^{4}\right) z^{2} \\
& +\left(1+q+2 q^{2}+2 q^{3}+2 q^{4}+q^{5}+q^{6}\right) z^{3}+\ldots,
\end{aligned}
$$

it follows that

$$
\frac{z+[1-(1+M(\theta))(q+1)] q z^{2}}{(1-z)(1-q z)\left(1-q^{2} z\right)}=z+\sum_{k=2}^{\infty}[k]_{q}\left(1-q M(\theta)[k-1]_{q}\right) a_{k} z^{k},
$$

where $M(\theta)$ is given by (2.2) and so (2.1) may be written as

$$
1-\sum_{k=2}^{\infty}[k]_{q} \frac{q[k-1]_{q}\left(e^{-i \theta}+B\right)-(A-B) b}{(A-B) b} a_{k} z^{k-1} \neq 0
$$

that is $(2.9)$.

Theorem 2.4. If $f(z) \in \mathcal{A}$, then $f(z) \in \mathcal{S}_{q}[b ; A, B]$ if and only if

$$
1-\sum_{k=2}^{\infty} \frac{\left([k]_{q}-1\right)\left(e^{-i \theta}+B\right)-(A-B) b}{(A-B) b} a_{k} z^{k-1} \neq 0 \text { for all } \theta \text {. }
$$


Proof. If $f(z) \in \mathcal{A}$, then from Theorem 2.2, we have $f(z) \in \mathcal{S}_{q}[b ; A, B]$ if and only if (2.7) holds. Since

$$
\frac{1}{(1-z)(1-q z)}=1+(1+q) z+\left(1+q+q^{2}\right) z^{2}+\left(1+q+q^{2}+q^{3}\right) z^{3}+\ldots,
$$

it follows that

$$
\frac{z-(1+M(\theta)) q z^{2}}{(1-z)(1-q z)}=z+\sum_{k=2}^{\infty}\left(1-q M(\theta)[k-1]_{q}\right) a_{k} z^{k},
$$

where $M(\theta)$ is given by (2.2). Now, we may express (2.7) as

$$
1-\sum_{k=2}^{\infty} \frac{q[k-1]_{q}\left(e^{-i \theta}+B\right)-(A-B) b}{(A-B) b} a_{k} z^{k-1} \neq 0,
$$

or equivalently, (2.10).

Theorem 2.5. If $f(z) \in \mathcal{A}$ satisfies the inequality

$$
\sum_{k=2}^{\infty}[k]_{q}\left[\left([k]_{q}-1\right)(1+|B|)+(A-B)|b|\right]\left|a_{k}\right| \leq(A-B)|b| .
$$

then $f(z) \in \mathcal{K}_{q}[b ; A, B]$.

Proof. Since

$$
\begin{aligned}
& \left|1-\sum_{k=2}^{\infty}[k]_{q} \frac{\left([k]_{q}-1\right)\left(e^{-i \theta}+B\right)-(A-B) b}{(A-B) b} a_{k} z^{k-1}\right| \\
& \geq 1-\left|\sum_{k=2}^{\infty}[k]_{q} \frac{\left([k]_{q}-1\right)\left(e^{-i \theta}+B\right)-(A-B) b}{(A-B) b} a_{k} z^{k-1}\right| \\
& \geq 1-\sum_{k=2}^{\infty}[k]_{q} \frac{\left([k]_{q}-1\right)(1+|B|)+(A-B)|b|}{(A-B)|b|}\left|a_{k}\right|>0 .
\end{aligned}
$$

Thus, the inequality (2.11) holds and our conclusion follows.

By using arguments and analysis to those in the proof of Theorem 2.5, we can analogously derive Theorem 2.6.

Theorem 2.6. If $f(z) \in \mathcal{A}$ satisfies

$$
\sum_{k=2}^{\infty}\left[\left([k]_{q}-1\right)(1+|B|)+(A-B)|b|\right]\left|a_{k}\right| \leq(A-B)|b| .
$$

then $f(z) \in \mathcal{S}_{q}[b ; A, B]$.

Remark 2.7. (i) For different choices of $q, b, A$ and $B$ in Theorems 2.1 and 2.2, the results of Seoudy and Aouf (see [20, Theorems 1 and 5]), Nasr and Aouf (see [14, Theorems 1 and 2]) and Bhoosnurmath and Devadas (see [6] and [7]) follow.

(ii) For $b=1$ in Theorems from 2.3 to 2.6, the results of Seoudy and Aouf (see [20, Theorems 9, 13, 17 and 21]) will follow. 
(iii) For different choices of $q, b, A$ and $B$ in our results, we will obtain new results for different classes mentioned in the introduction.

\section{References}

[1] Abu-Risha, M.H., Annaby, M.H., Ismail, M.E.H., Mansour, Z.S., Linear q-difference equations, Z. Anal. Anwend., 26(2007), 481-494.

[2] Aouf, M.K., Bounded spiral-like functions with fixed second coefficient, Int. J. Math. Math. Sci., 12(1989), 113-118.

[3] Aouf, M.K., Darwish, H.E., Attiya, A.A., Coefficient estimates for certain classes of analytic functions, Punjab Univ. J. Math., 33(2000), 51-64.

[4] Aouf, M.K., Darwish, H.E., Attiya, A.A., Coefficients estimates for certain class of analytic functions with complex order, Math. Sci. Res. Hot-Line, 2(11)(1998), 1-13.

[5] Aouf, M.K., Mostafa, A.O., Zayed, H.M., Convolution properties for some subclasses of meromorphic functions of complex order, Abstr. Appl. Anal., 2015(2015), Art. ID 973613, 6 pages.

[6] Bhoosnurmath, S.S., Devadas, M.V., Subclasses of spirallike functions defined by subordination, J. Anal., 4(1996), 173-183.

[7] Bhoosnurmath, S.S., Devadas, M.V., Subclasses of spirallike functions defined by Ruschweyh derivatives, Tamkang J. Math., 28(1997), 59-65.

[8] Dashrath, Shukla, S.L., Coefficient estimates for a subclass of spirallike functions, Indian J. Pure Appl. Math., 14(1983), 431-439.

[9] Gasper, G., Rahman, M., Basic Hypergeometric Series, Cambridge University Press, Cambridge, 1990.

[10] Jakubowski, Z.J., On the coefficients of starlike functions of some classes, Ann. Polon. Math., 26(1972), 305-313.

[11] Kac, V.G., Cheung, P., Quantum Calculus, Universitext, Springer-Verlag, New York, 2002.

[12] Kulshrestha, P.K., Bounded Robertson functions, Rend. Mat. (Ser. 6), 9(1976), 137-150.

[13] Miller, S.S., Mocanu, P.T., Differential Subordinations: Theory and Applications, Series on Monographs and Textbooks in Pure and Applied Mathematics, No. 255, Marcel Dekker, New York, 2000.

[14] Nasr, M.A., Aouf, M.K., Characterizations for convex functions and starlike functions of complex order in $\mathbb{U}=\{z:|z|<1\}$, Bull. Fac. Sci. Assiut Univ., 11(1982), 117-121.

[15] Nasr, M.A., Aouf, M.K., On convex functions of complex order, Bull. Fac. Sci. Mansoura Univ., 9(1982), 565-582.

[16] Nasr, M.A., Aouf, M.K., Bounded starlike functions of complex order, Proc. Indian Acad. Sci. Math. Sci., 92(1983), 97-102.

[17] Nasr, M.A., Aouf, M.K., Bounded convex functions of complex order, Bull. Fac. Sci. Mansoura Univ., 10(1983), 513-527.

[18] Nasr, M.A., Aouf, M.K., Starlike function of complex order, J. Natur. Sci. Math., 25(1985), 1-12.

[19] Rajković, P.M., Marinković, S.D., Stanković, M.S., Fractional integrals and derivatives in q-calculus, Appl. Anal. Discrete Math., 1(2007), 311-323. 
[20] Seoudy, T.M., Aouf, M.K., Convolution properties for certain classes of analytic functions defined by q-derivative operator, Abstr. Appl. Anal., 2014(2014), Art. ID 846719, $1-7$.

[21] Sohi, N.S., Singh, L.P., A class of bounded starlike functions of complex order, Indian J. Math., 33(1991), 29-35.

[22] Srivastava, H.M., Univalent functions, fractional calculus, and associated generalized hypergeometric functions, in Univalent Functions, Fractional Calculus, and Their Applications (H. M. Srivastava and S. Owa, Editors), Halsted Press (Ellis Horwood Limited, Chichester), pp. 329-354, John Wiley and Sons, New York, Chichester, Brisbane and Toronto, 1989.

[23] Srivastava, H.M., Aouf, M.K., Mostafa, A.O., Zayed, H.M., Certain subordinationpreserving family of integral operators associated with $p$ valent functions, Appl. Math. Inf. Sci., 11(2017), no. 4, 951-960.

[24] Srivastava, H.M., Bansal, D., Close-to-convexity of a certain family of q-Mittag-Leffler functions, J. Nonlinear Var. Anal., 1(2017), no. 1, 61-69.

[25] Srivastava, H.M., Owa, S. (eds.), Current Topics in Analytic Function Theory, World Scientific Publishing Company, Singapore, New Jersey, London, and Hong Kong, 1992.

[26] Xu, Q.-H., Gui, Y.-C., Srivastava, H.M., Coefficient estimates for certain subclasses of analytic functions of complex order, Taiwanese J. Math., 15(2011), 2377-2386.

Rekha Srivastava

Department of Mathematics and Statistics

University of Victoria

Victoria, British Columbia V8W 3R4, Canada

e-mail: rekhas@math.uvic.ca

Hanaa M. Zayed

Department of Mathematics

Faculty of Science

Menofia University, Shebin Elkom 32511, Egypt

e-mail: hanaa_zayed42@yahoo.com 